\title{
Two ways to generate monotonic sequences: convexity and ratios
}

G. J. O. Jameson

\section{Introduction}

Here we give an account of two appealingly simple techniques to generate monotonic sequences that were developed by the author and Grahame Bennett. Sadly, Bennett died in 2016 after a lifetime of distinguished contributions in the field of inequalities. This article is dedicated to him.

The first method works with averages of values of convex functions. It was described in full in [1]; a partial version was given by Kuang in a Gazette note [2]. The second method deals with ratios of partial sums. The idea has been known for a long time, but the applications described here were first presented in [3], as part of a wider study.

The main application relates to the sum $S_{n}(p)=\sum_{r=1}^{n} r^{p}$. We present a companion pair of results on monotonicity of $S_{n}(p) /\left[n^{p}(n+1)\right]$ and $S_{n}(p) /\left[n(n+1)^{p}\right]$ for various values of $p$. At first sight, these ratios may seem a bit abstruse, but in a sense they are more natural than, for example, $S_{n}(p) / n^{p+1}$, because they reproduce the fact that $S_{n}(1) /[n(n+1)]$ is constant. We show how these results can be obtained equally by both methods. Another result delivered by both methods is a strengthening of the theorem of [4] concerning $(n !)^{1 / n}$.

We then present a result on sums of the form $\sum_{r=1}^{n}(2 r-1)^{p}$ which is delivered by Method 2 but not at all easily by Method 1. We finish with a survey of various other averages of convex functions.

\section{Method 1: averages of convex functions}

For a function $f$ on the interval $[0,1]$, we define

$$
\begin{array}{ll}
A_{n}(f)=\frac{1}{n-1} \sum_{r=1}^{n-1} f\left(\frac{r}{n}\right) & (n \geq 2), \\
B_{n}(f)=\frac{1}{n+1} \sum_{r=0}^{n} f\left(\frac{r}{n}\right) & (n \geq 1) .
\end{array}
$$

These are, respectively, the averages of the values $f\left(\frac{r}{n}\right)$ excluding and including the end points. For $A_{n}(f)$, we do not need $f$ to be defined at 0 and 1 . If $f$ is continuous on $[0,1]$, then both $A_{n}(f)$ and $B_{n}(f)$ tend to $\int_{0}^{1} f(x) d x$ as $n \rightarrow \infty$ (we return to this point later). The point for us is that for convex functions, they do so in a monotonic way. 
Recall that $f$ is "convex" on an interval $I$ if it lies below the straight-line chord between any two points on its graph. More exactly, for $x_{1}, x_{2}$ in $I$ and $0 \leq \lambda \leq 1$, we have $f[(1-\lambda) x+\lambda y] \leq(1-\lambda) f(x)+\lambda f(y)$. Also, $f$ is "concave" if $-f$ is convex. If a function is both convex and concave, then it is linear. A sufficient condition for convexity is that $f^{\prime \prime}(x) \geq 0$ on the interval. So for $x>0$, the function $x^{p}$ is convex for $p \geq 1$ and $p \leq 0$, and concave for $0 \leq p \leq 1$. We can now state our results.

Theorem 1: If $f$ is a convex function on $(0,1)$, then $A_{n}(f)$ increases with $n$. If $f$ is concave, then $A_{n}(f)$ decreases with $n$.

Proof: We prove the statement for convex $f$; the statement for concave $f$ then follows by considering $-f$. For $1 \leq r \leq n-1$, the point $r / n$ lies between $r /(n+1)$ and $(r+1) /(n+1)$. More exactly,

$$
\frac{r}{n}=\frac{n-r}{n} \frac{r}{n+1}+\frac{r}{n} \frac{r+1}{n+1}
$$

Write $f[r /(n+1)]=f_{r}$. Since $f$ is convex,

$$
f\left(\frac{r}{n}\right) \leq \frac{n-r}{n} f_{r}+\frac{r}{n} f_{r+1}
$$

Hence

$$
\begin{aligned}
\sum_{r=1}^{n-1} f\left(\frac{r}{n}\right) & \leq \frac{n-1}{n} f_{1}+\frac{1}{n} f_{2}+\frac{n-2}{n} f_{2}+\frac{2}{n} f_{3}+\cdots+\frac{1}{n} f_{n-1}+\frac{n-1}{n} f_{n} \\
& =\frac{n-1}{n} \sum_{r=1}^{n} f_{r}
\end{aligned}
$$

which says that $A_{n}(f) \leq A_{n+1}(f)$.

Theorem 2: If $\mathrm{f}$ is convex on $[0,1]$, then $B_{n}(f)$ decreases with $n$. If $\mathrm{f}$ is concave, then $B_{n}(f)$ increases with $n$.

Proof: Assume $f$ convex and take $n \geq 2$. This time, we use the fact that for $1 \leq r \leq$ $n-1$,

$$
\frac{r}{n}=\frac{r}{n} \frac{r-1}{n-1}+\frac{n-r}{n} \frac{r}{n-1}
$$

Write $f[r /(n-1)]=g_{r}$. By convexity of $f$, for $r$ as above,

$$
f\left(\frac{r}{n}\right) \leq \frac{r}{n} g_{r-1}+\frac{n-r}{n} g_{r}
$$

also $f(0 / n)=g_{0}$ and $f(n / n)=g_{n-1}$. Hence

$$
\begin{aligned}
\sum_{r=0}^{n} f\left(\frac{r}{n}\right) & \leq \frac{n}{n} g_{0}+\frac{1}{n} g_{0}+\frac{n-1}{n} g_{1}+\frac{2}{n} g_{1}+\cdots+\frac{1}{n} g_{n-1}+\frac{n}{n} g_{n-1} \\
& =\frac{n+1}{n} \sum_{r=0}^{n-1} g_{r},
\end{aligned}
$$


which says that $B_{n}(f) \leq B_{n-1}(f)$.

Of course, if $f$ is linear, it follows that $A_{n}(f)$ and $B_{n}(f)$ are constant. This is easily verified directly: if $f(x)=x$, then $A_{n}(f)=B_{n}(f)=\frac{1}{2}$ for all $n$.

\section{Method 2: ratios of partial sums}

Our second method for generating monotonic sequences is beautifully simple both to state and to prove. It is far from new: it appears as an exercise (albeit without serious applications) in [5, p. 21], published in 1908.

Theorem 3: Let $\left(a_{n}\right)$ and $\left(b_{n}\right)$ be sequences, with $b_{n}>0$ for all $n$. Write $A_{n}=$ $a_{1}+a_{2}+\cdots+a_{n}$, similarly $B_{n}$. If $a_{n} / b_{n}$ increases (or decreases) with $n$, so does $A_{n} / B_{n}$.

(Of course, this notation $A_{n}$ has no connection with the previous $A_{n}(f)$.)

Proof: We prove the increasing case: the decreasing one follows by considering $-a_{n}$. Write $a_{n}=c_{n} b_{n}$ and $A_{n}=K_{n} B_{n}$. Then $\left(c_{n}\right)$ is increasing, and hence $A_{n}=\sum_{r=1}^{n} c_{r} b_{r} \leq$ $c_{n} \sum_{r=1}^{n} b_{r}=c_{n} B_{n}$, so $K_{n} \leq c_{n}$. So also $K_{n} \leq c_{n+1}$, and we have

$$
\begin{aligned}
A_{n+1} & =A_{n}+a_{n+1} \\
& =K_{n} B_{n}+c_{n+1} b_{n+1} \\
& \geq K_{n}\left(B_{n}+b_{n+1}\right) \\
& =K_{n} B_{n+1} .
\end{aligned}
$$

But $A_{n+1}=K_{n+1} B_{n+1}$, so $K_{n+1} \geq K_{n}$.

The special case $b_{n}=1$ says: if $a_{n}$ is increasing, then so is $A_{n} / n$, the arithmetic mean of $a_{1}, a_{2}, \ldots, a_{n}$. We mention here that we can deduce the same for the geometric mean:

Corollary 3.1: Let $\left(a_{n}\right)$ be an increasing sequence of positive numbers, and let $G_{n}=$ $\left(a_{1} a_{2} \ldots a_{n}\right)^{1 / n}$. Then $\left(G_{n}\right)$ increases with $n$. In particular, $(n !)^{1 / n}$ is increasing.

Proof: Since $\log a_{n}$ increases with $n$, so does $\frac{1}{n} \sum_{r=1}^{n} \log a_{r}=\log G_{n}$.

Of course, to apply Theorem 3 , we have to equip ourselves with the sequence $a_{n} / b_{n}$. The examples to follow will illustrate what this may involve.

\section{Application to $S_{n}(p)$}

Let $S_{n}(p)=\sum_{r=1}^{n} r^{p}$. We will show how both methods can be applied to this sum, with a similar outcome. Note that $S_{n}(0)=n$ and $S_{n}(1)=\frac{1}{2} n(n+1)$. We do not exclude 
negative values of $p$ : for example, $S_{n}(-1)$ is the harmonic sum $H_{n}=\sum_{r=1}^{n} \frac{1}{r}$.

A basic estimation of $S_{n}(p)$ (which we will shortly improve) is given by comparison with the integral of $x^{p}$, as follows. Let $f$ be any increasing function with $f(0)=0$. Write $S_{n}=\sum_{r=1}^{n} f(r)$. On the interval $[r-1, r]$, we have $f(r-1) \leq f(x) \leq f(r)$, hence $f(r-1) \leq$ $\int_{r-1}^{r} f(x) d x \leq f(r)$. Adding the right-hand inequalities for $1 \leq r \leq n$ and the left-hand inequalities for $1 \leq r \leq n+1$, we obtain $\int_{0}^{n} f(x) d x \leq S_{n} \leq \int_{0}^{n+1} f(x) d x$. For $f(x)=x^{p}$, where $p>0$, this says

$$
\frac{n^{p+1}}{p+1} \leq S_{n}(p) \leq \frac{(n+1)^{p+1}}{p+1}
$$

hence $S_{n}(p) / n^{p+1} \rightarrow 1 /(p+1)$ as $n \rightarrow \infty$. In fact, this limit also holds for $-1<p<0$ (we omit the details). Of course, for $p<-1, S_{n}(p)$ is simply the $n$th partial sum of the convergent series $\sum_{r=1}^{\infty} r^{p}$.

We are now ready for our Theorem on monotonicity.

Theorem 4: Let

$$
c_{n}(p)=\frac{S_{n}(p)}{n(n+1)^{p}} .
$$

Then $c_{n}(p)$ increases with $n$ for $p \geq 1$ and $p \leq 0$, and decreases for $0 \leq p \leq 1$.

Also, let

$$
d_{n}(p)=\frac{S_{n}(p)}{n^{p}(n+1)} .
$$

Then $d_{n}(p)$ decreases with $n$ for $p \geq 1$, and increases for $p \leq 1$.

The reversals at $p=1$ reflect the fact that $c_{n}(1)$ and $d_{n}(1)$ have the constant value $\frac{1}{2}$. Similarly, $c_{n}(0)$ has the constant value 1 .

Ahead of the proof (by both methods), note that for $p \leq 0$, the statement for $d_{n}(p)$ follows from the one for $c_{n}(p)$. In fact,

$$
d_{n}(p)=\left(\frac{n+1}{n}\right)^{p-1} c_{n}(p)
$$

Now $(n+1) / n=1+\frac{1}{n}$, which is decreasing. Since $p-1<0,[(n+1) / n]^{p-1}$ is increasing.

Recall that $x^{p}$ is convex for $p \geq 1$ and $p \leq 0$, and concave for $0 \leq p \leq 1$. With this remark, we can read off Theorem 4 from Theorems 1 and 2 without further work.

Proof by Method 1: The statements for $c_{n}(p)$ are given by Theorem 1 with $f(x)=x^{p}$, since

$$
A_{n+1}(f)=\frac{1}{n} \sum_{r=1}^{n} \frac{r^{p}}{(n+1)^{p}}=c_{n}(p) .
$$


By the preceding remark, we only need to consider $d_{n}(p)$ for $p \geq 0$. The statements are given by Theorem 2 , since

$$
B_{n}(f)=\frac{1}{n+1} \sum_{r=0}^{n} \frac{r^{p}}{n^{p}}=d_{n}(p) .
$$

The proof by Method 2 needs a little more work. We include it for comparison, and because it paves the way for the proof of another result to follow.

Proof by Method 2: We apply Theorem 3 to $1 / c_{n}(p)$ : this equals $A_{n} / B_{n}$, where

$$
a_{n}=n(n+1)^{p}-(n-1) n^{p}, \quad b_{n}=n^{p} .
$$

So for $p \neq 0$,

$$
\frac{a_{n}}{b_{n}}=n\left(1+\frac{1}{n}\right)^{p}-n+1
$$

Now

$$
\frac{d}{d x}\left(1+\frac{x}{n}\right)^{p}=\frac{p}{n}\left(1+\frac{x}{n}\right)^{p-1}
$$

and hence

$$
\frac{a_{n}}{b_{n}}=n\left[\left(1+\frac{x}{n}\right)^{p}\right]_{0}^{1}+1=p \int_{0}^{1}\left(1+\frac{x}{n}\right)^{p-1} d x+1 .
$$

Now $1+\frac{x}{n}$ decreases with $n$, so $\left(1+\frac{x}{n}\right)^{p-1}$, and hence its integral, decreases if $p \geq 1$ and increases if $p<1$. For $a_{n} / b_{n}$, this is reversed by the factor $p$ when $p<0$. The result follows.

For $d_{n}(p)$, with $p>0$, we have $1 / d_{n}(p)=A_{n} / B_{n}$, where

$$
a_{n}=n^{p}(n+1)-(n-1)^{p} n, \quad b_{n}=n^{p} .
$$

So, in similar style,

$$
\frac{a_{n}}{b_{n}}=n+1-n\left(1-\frac{1}{n}\right)^{p}=1+p \int_{0}^{1}\left(1-\frac{x}{n}\right)^{p-1} d x .
$$

This increases with $n$ if $p \geq 1$ and decreases if $0<p<1$.

Example 1: We can illustrate the case $p=2$ by giving exact expressions. Since $S_{n}(2)=$ $\frac{1}{6} n(n+1)(2 n+1)$, we have

$$
6 c_{n}(2)=2-\frac{1}{n+1}, \quad 6 d_{n}(2)=2+\frac{1}{n},
$$

showing that $c_{n}(2)$ is indeed increasing and $d_{n}(2)$ decreasing.

Example 2: The case $p=-1$ for $c_{n}(p)$ says that $\left(1+\frac{1}{n}\right) H_{n}$ increases with $n$. On writing it out, one finds that this actually follows from the inequality $H_{n} \leq n$. 
We mention a number of consequences of Theorem 4 .

If $p>0$, it follows from $(1)$ that $c_{n}(p)$ and $d_{n}(p)$ tend to $1 /(p+1)$ as $n \rightarrow \infty$. Since the terms of an increasing sequence are not greater than the limit, we can deduce the following refinement of (1):

Corollary 4.1: For $p \geq 1$, we have

$$
\frac{1}{p+1} n^{p}(n+1) \leq S_{n}(p) \leq \frac{1}{p+1} n(n+1)^{p} .
$$

The inequalities reverse when $0<p \leq 1$.

At the cost of some loss of accuracy, we can deduce simpler statements where the denominators are $n^{p+1}$ and $(n+1)^{p+1}$.

Corollary 4.2: Let $u_{n}(p)=S_{n}(p) / n^{p+1}$. Then $u_{n}(p)$ decreases if $p \geq 0$ and increases if $p \leq 0$.

Proof: We have $u_{n}(p)=\left(1+\frac{1}{n}\right) d_{n}(p)$, hence is decreasing for $p \geq 1$. Also, $u_{n}(p)=$ $\left(1+\frac{1}{n}\right)^{p} c_{n}(p)$, hence is decreasing for $0 \leq p \leq 1$ and increasing for $p<0$.

Corollary 4.3: Let $v_{n}(p)=S_{n}(p) /(n+1)^{p+1}$. Then $v_{n}(p)$ is increasing for all $p$.

Proof: For $p \geq 1$ and $p<0$, this follows from $v_{n}(p)=[n /(n+1)] c_{n}(p)$, since $n /(n+1)$ is increasing. For $0<p \leq 1$, it follows from $v_{n}(p)=\left[n /(n+1]^{p} d_{n}(p)\right.$.

Note that unlike $c_{n}(p)$ and $d_{n}(p)$, these ratios fail to be constant when $p=1$.

\section{Application to $n$ !}

We now describe another result delivered by both methods.

Theorem 5: The expression $\frac{1}{n+1}(n !)^{1 / n}$ decreases with $n$.

It was shown in [4] that $\frac{1}{n}(n !)^{1 / n}$ decreases; this statement is weaker than ours, because their expression equates to ours multiplied by the decreasing factor $1+\frac{1}{n}$.

Proof 1: We apply Theorem 1 to the concave function $\log x$ (recall that for this Theorem there is no need for $f$ to be defined at 0$)$. Then $A_{n+1}(f)$ decreases with $n$. The result follows, since

$$
A_{n+1}(f)=\frac{1}{n} \sum_{r=1}^{n}(\log r-\log (n+1))=\frac{1}{n} \log (n !)-\log (n+1) .
$$

Proof 2: Let $a_{n}=n[\log (n+1)-\log n]$. Then $a_{n}=\int_{0}^{1} \frac{n}{n+x} d x$. For $x \geq 0, \frac{n}{n+x}$ increases 
with $n$, since $\frac{n+x}{n}=1+\frac{x}{n}$ decreases. So $a_{n}$ increases. By Theorem 3 , so does $A_{n} / n$. But

$$
\frac{A_{n}}{n}=\frac{1}{n} \sum_{r=1}^{n} r[\log (r+1)-\log r]=\log (n+1)-\frac{1}{n} \sum_{r=1}^{n} \log r .
$$

Note: There is another application of Proof 2: take $a_{n}=\log (n+1)-\log n$ and $b_{n}=\frac{1}{n}$, to conclude that $\log (n+1) / H_{n}$ increases with $n$.

\section{Some further applications of Method 2}

Method 1 gives an instant proof for sequences that are of one of the forms described, but it is limited to such sequences. Method 2 is effective in some areas beyond the reach of Method 1. One such area concerns odd-number sums. Let

$$
U_{n}(p)=\sum_{r=1}^{n}(2 r-1)^{p}
$$

An immediate application of Theorem 3 is that $U_{n}(p) / S_{n}(p)$ increases with $n$ for $p>0$, since this is of the form $A_{n} / B_{n}$, where $a_{n} / b_{n}=\left(2-\frac{1}{n}\right)^{p}$. More seriously, by a suitable modification of the proof by Method 2, we can derive a pleasantly simple analogue of Theorem 4 for $U_{n}(p)$. This proof follows [2, p. 559].

Theorem 6: The expression $\frac{U_{n}(p)}{n^{p+1}}$ increases with $n$ if $p \geq 1$ or $p \leq 0$, and decreases if $0 \leq p \leq 1$

Remark: The reversal at $p=1$ reflects the fact that $U_{n}(1)=n^{2}$.

Lemma: Let $g(t)=(1+t)^{p}+(1-t)^{p}$. If $p \geq 1$ or $p \leq 0$, then $g(t)$ increases with $t$ for $0 \leq t<1$. If $0 \leq p \leq 1$, then $g(t)$ decreases.

Proof: We have $g^{\prime}(t)=p\left[(1+t)^{p-1}-(1-t)^{p-1}\right]$. Hence $g^{\prime}(t) \geq 0$ on $[0,1)$ if $p \geq 1$ or $p \leq 0$, and $g^{\prime}(t) \leq 0$ if $0 \leq p \leq 1$.

Proof of Theorem 6: If $p \leq-1$, then $1 / n^{p+1}=n^{q}$ where $q \geq 0$, so increases with $n$, so the statement is trivial. So assume that $p>-1$. We have $n^{p+1} / U_{n}(p)=A_{n} / B_{n}$, where

$$
a_{n}=n^{p+1}-(n-1)^{p+1}, \quad b_{n}=(2 n-1)^{p} .
$$

Then

$$
\begin{aligned}
\frac{a_{n}}{b_{n}} & =(2 n-1)\left[\left(\frac{n}{2 n-1}\right)^{p+1}-\left(\frac{n-1}{2 n-1}\right)^{p+1}\right] \\
& =\frac{2 n-1}{2^{p+1}}\left[\left(1+\frac{1}{2 n-1}\right)^{p+1}-\left(1-\frac{1}{2 n-1}\right)^{p+1}\right] .
\end{aligned}
$$


Now

$$
(2 n-1) \frac{d}{d x}\left(1+\frac{x}{2 n-1}\right)^{p+1}=(p+1)\left(1+\frac{x}{2 n-1}\right)^{p}
$$

so

$$
\frac{a_{n}}{b_{n}}=\frac{p+1}{2^{p+1}} I_{n}
$$

where

$$
I_{n}=\int_{-1}^{1}\left(1+\frac{x}{2 n-1}\right)^{p} d x=\int_{0}^{1}\left[\left(1+\frac{x}{2 n-1}\right)^{p}+\left(1-\frac{x}{2 n-1}\right)^{p}\right] d x .
$$

By the Lemma, $I_{n}$ decreases with $n$ if $p \geq 1$ or $p \leq 0$, and increases with $n$ if $0 \leq p \leq 1$. The statement follows, by Theorem 3 .

Clearly, this sum is not a case of $A_{n}(f)$ or $B_{n}(f)$. In the next section, we will see what happens when this problem is addressed by function averages.

By a slight variation of the proof of Theorem 6, one can establish another result of this kind for $S_{n}(p)$ : the expression

$$
\frac{S_{n}(p)}{(2 n+1)^{p+1}-1}
$$

increases with $n$ if $p \geq 1$ or $-1<p \leq 0$, and decreases if $0 \leq p \leq 1$. The reader may care to attempt the details. Note that again the expression is constant when $p=1$, since $(2 n+1)^{2}-1=4 n(n+1)$.

\section{Some other averages of convex functions}

First, we investigate averages including one end-point. Write

$$
L_{n}(f)=\frac{1}{n} \sum_{r=0}^{n-1} f\left(\frac{r}{n}\right), \quad R_{n}(f)=\frac{1}{n} \sum_{r=1}^{n} f\left(\frac{r}{n}\right) .
$$

Simple formulae link these sums with $A_{n}(f)$ and $B_{n}(f)$, for example $n R_{n}(f)=\sum_{r=1}^{n} f\left(\frac{r}{n}\right)=$ $(n+1) B_{n}(f)-f(0)$, hence $R_{n}(f)=\left(1+\frac{1}{n}\right) B_{n}(f)-\frac{1}{n} f(0)$. Note also that if $g(x)=f(1-x)$, then $L_{n}(g)=R_{n}(f)$.

These unsymmetrical averages are arguably less natural than $A_{n}(f)$ and $B_{n}(f)$. They fail to be constant for $f(x)=x$ : in fact, then $L_{n}(f)=\frac{1}{2}\left(1-\frac{1}{n}\right)$ and $R_{n}(f)=\frac{1}{2}\left(1+\frac{1}{n}\right)$. So $R_{n}(f)$ is decreasing for $f(x)=x$, but increasing for $f(x)=-x$ : there is no result to the effect that $R_{n}(f)$ is either increasing or decreasing for all convex $f$. However, restricted results on monotonicity do follow easily from Theorems 1 and 2:

Theorem \%: If $f$ is convex and increasing, then $R_{n}(f)$ decreases with $n$ and $L_{n}(f)$ increases. If $f$ is convex and decreasing, then $R_{n}(f)$ increases and $L_{n}(f)$ decreases. 
Proof: The statements for $L_{n}(f)$ follow from those for $R_{n}(f)$ by applying them to $g(x)=f(1-x)$. Suppose that $f$ is convex and increasing. Let $h(x)=f(x)-f(0)$. Then $R_{n}(h)=R_{n}(f)-f(0), \quad h(0)=0$ and $h(x) \geq 0$ on $[0,1]$. So $R_{n}(h)=\left(1+\frac{1}{n}\right) B_{n}(h)$ and $B_{n}(h) \geq 0$. By Theorem $2, B_{n}(h)$ is decreasing. Hence $R_{n}(h)$ is decreasing.

Similarly, if $f$ is decreasing, let $k(x)=f(x)-f(1)$. Then $R_{n}(k)=\left(1-\frac{1}{n}\right) A_{n}(k)$, which is increasing, by Theorem 1 .

This result was presented in [3]. While it serves to satisfy curiosity about averages with one end point, it is clear that it is weaker than Theorems 1 and 2 .

Applied to $f(x)=x^{p}$, Theorem 7 reproduces Corollaries 4.2 and (for $p>0$ ) 4.3, since $u_{n}(p)=R_{n}(f)$ and $v_{n}(p)=L_{n+1}(f)$.

We can now clarify the relation with $\int_{0}^{1} f(x) d x$. If $f$ is continuous on $[0,1]$, then $L_{n}(f)$ and $R_{n}(f)$ are Riemann sums for this integral (in the sense that a value of $f(x)$ is chosen from each successive interval). So they both converge to the integral as $n \rightarrow \infty$, and it follows that the same is true for $A_{n}(f)$ and $B_{n}(f)$, as stated earlier.

We now revisit Theorem 6 . The sum is of the form

$$
M_{n}(f)=\frac{1}{n} \sum_{r=1}^{n} f\left(\frac{2 r-1}{2 n}\right) .
$$

What can we say about this kind of average? It passes the test of being constant for $f(x)=x$. It describes the approximation to $\int_{0}^{1} f(x) d x$ given by taking the tangent to the curve at the mid-point of each interval $\left[\frac{r-1}{n}, \frac{r}{n}\right]$. A convex function $f$ lies above its tangents, so $M_{n}(f) \leq \int_{0}^{1} f(x) d x$.

A dual approximation to the integral is given by the trapezium rule: let

$$
T_{n}(f)=\frac{1}{2 n} f(0)+\frac{1}{n} \sum_{r=1}^{n-1} f\left(\frac{r}{n}\right)+\frac{1}{2 n} f(1) .
$$

From the definition of convexity, it is clear that $T_{n}(f) \geq \int_{0}^{1} f(x) d x$ for convex functions.

In the light of these facts, it seems plausible that for convex $f, M_{n}(f)$ increases with $n$ and $T_{n}(f)$ decreases. However, this is not true, as the following example shows.

Example 3: Let $f(x)=\left|x-\frac{1}{2}\right|$. Then $M_{2}(f)=\frac{1}{2}\left(\frac{1}{4}+\frac{1}{4}\right)=\frac{1}{4}$, while $M_{3}(f)=\frac{1}{3}\left(\frac{1}{3}+0+\right.$ $\left.\frac{1}{3}\right)=\frac{2}{9}$. The reader can easily verify that $T_{2}(f)=\frac{1}{4}$ and $T_{3}(f)=\frac{5}{18}$.

The true state of affairs was established in [1]: the suspected results hold if $f$ is convex and also $f^{\prime}$ is either convex or concave. This, of course, delivers Theorem 6 . However, the 
proofs given in [1] are long and intricate. A more pleasant proof of both results is presented in the forthcoming article [6].

A natural generalisation of $A_{n}(f)$ and $B_{n}(f)$ is a weighted average of the form $B_{n}(W, f)=$ $\sum_{r=0}^{n} w_{n, r} f\left(\frac{r}{n}\right)$, where $w_{n, r} \geq 0$ and $\sum_{r=0}^{n} w_{n, r}=1$ for all $n$. This is explored in [7]. For example, it is shown that $B_{n}(W, f)$ decreases with $n$ for convex $f$ when $W$ is the Euler matrix defined by $w_{n, r}=\left(\begin{array}{l}n \\ r\end{array}\right) x^{r}(1-x)^{n-r}$ for a chosen $x$ in $(0,1)$. As some readers will recognise, $B_{n}(W, f)$ is then the value at $x$ of the $n$th Bernstein polynomial for $f$.

\section{References}

[1] G. Bennett and G. J. O. Jameson, Monotonic averages of convex functions, J. Math. Anal. Appl. 252 (2000), 410-430.

[2] G. Bennett, Meaningful sequences, Houston J. Math. 33 (2007), 555-580.

[3] Kuang Jichang, Some extensions and refinements of Minc-Sathre inequality, Math. Gazette 83 (1999), 123-127.

[4] H. Minc and L. Sathre, Some inequalities concerning $(r !)^{1 / r}$, Proc. Edinburgh Math. Soc. 14 (1964), 41-46.

[5] T. J. I'a. Bromwich, An Introduction to the Theory of Infinite Series, 1st ed., MacMillan (1908).

[6] G. J. O. Jameson, Monotonicity of the mid-point and trapezium estimates for integrals, Math. Gazette 105 (2021), to appear.

[7] G. J. O. Jameson, Monotonicity of weighted averages of convex functions, Math. Ineq. Appl., 23 (2020), 425-432.

13 Sandown Road, Lancaster LA1 4LN, UK e-mail: pgjameson@talktalk.net 